\title{
Epigenetic Regulation of Stem Cell Differentiation
}

\author{
HAO WU, AND YI EVE SUN \\ Departments of Molecular and Medical Pharmacology and Psychiatry and Behavioral Sciences, Neuropsychiatric Institute, University of \\ California, Los Angeles, School of Medicine, Los Angeles, CA 90095
}

\begin{abstract}
Stem cells undergo extensive self-renewal and have the capacity to differentiate along multiple cell lineages. Progression from stem cells into differentiated progeny requires long-lasting changes in gene expression. Epigenetic mechanisms, including DNA methylation, histone modifications, and non-coding RNA-mediated regulatory events, are essential to controlling the heritable cellular memory of gene expression during development. Recent studies on cell fate specification of embryonic and adult stem cells/progenitors have highlighted a general and critical role for dynamic epigenetic regulation in stem cell self-renewal and differentiation. (Pediatr Res 59: 21R-25R, 2006)
\end{abstract}

$\mathrm{S}$ em cells are characterized by at least two essential properties: extensive self-renewal and the ability to differentiate into different cell-type specific progenies. Embryonic stem cells (ESCs) and primordial germ cells are pluripotent stem cells, both of which can give rise to essentially all cell types within a bioorganism. Tissue-specific stem/progenitor cells persist in many, if not all, tissues throughout life. These stem/progenitor cells are usually multipotent or unipotent, and can differentiate into lineage-restricted progenies. Intrinsic properties of stem cells as well as extrinsic cues provided by the niche (microenvironment where stem cells reside in vivo) are important for stem cell selfrenewal and differentiation. Progression from stem cell to differentiated progeny is almost always accompanied by remarkable changes in cellular morphology and function. To a large extent, these changes are determined at each stage by distinctive gene expression patterns. Specifically, genes associated with self-renewal are silenced, while cell type-specific genes undergo transcriptional activation during differentiation. Emerging evidence suggests that the initiation and maintenance of changes in gene expression that are associated with stem cell differentiation involve the action of a unique epigenetic program including covalent DNA and chromatin modifications as well as small noncoding RNA-mediated pre- and posttranscriptional gene regulations.

\section{MAJOR EPIGENETIC MECHANISMS}

Epigenetic mechanisms refer to biologic processes that regulate mitotically or meiotically, heritable changes in gene expression without altering the DNA sequence. Major epige-

Received December 8, 2005; accepted December 8, 2005.

Correspondence: Yi Eve Sun, Ph.D., Departments of Molecular \& Medical Pharmacology and Psychiatry \& Behavioral Sciences, UCLA School of Medicine, 635 Charles E. Young Drive South, NRB room 351, Los Angeles, CA 90095; e-mail: ysun@mednet.ucla.edu.

This work was supported by National Institutes of Health RO1 grants to Y.E.S., and by a Beckman Young Investigator Award and an Alfred Sloan Fellowship to Y.E.S.

DOI: $10.1203 / 01 . p d r .0000203565 .76028 .2 \mathrm{a}$ netic mechanisms include DNA cytosine methylation, histone modifications such as acetylation and methylation of histone tails, and small non-coding RNA controlled pre- and posttranscriptional regulation of gene expression.

DNA Methylation. Mammalian DNA can be covalently modified through methylation of the carbon at the 5th position on the pyrimidine ring of the cytosine residue. In mammals, DNA methylation occurs primarily at symmetrical $\mathrm{CpG}$ dinucleotides. De novo cytosine methylation, catalyzed by two de novo DNA methyltransferases, Dnmt3a and Dnmt3b, adds methyl-groups onto unmethylated DNA, which is important for the establishment of DNA methylation patterns. Upon cell division, the existing methylation patterns need to be maintained by perhaps a more efficient DNA methyltransferase, the maintenance methyltransferase Dnmt1, which prefers hemimethylated substrates (Fig. 1). DNA methylation is primarily involved in establishing parental-specific imprinting during gametogenesis as well as silencing of retrotransposons and genes on the inactivated X-chromosome $(1,2)$.

Histone modifications. Post-translational modifications of histones, including acetylation and methylation of conserved lysine residues on the amino terminal tail, are also dynamically regulated by chromatin modifying enzymes with opposing activities. The idea of two opposing activities, leading to two opposing states on the chromatin underlies the "histone code" hypothesis (3). Generally, lysine acetylation mediated by histone acetyltransferases (HATs) marks transcriptionally competent regions. In contrast, histone deacetylases (HDACs) catalyze lysine deacetylation and the resulting hypoacetylated histones are usually associated with transcriptionally inactive chromatin structures. Histone methylation of lysine 4 on histone $\mathrm{H} 3$ (H3-K4) is generally present within transcriptionally competent promoter regions. Furthermore, the lysine residues can be mono-, di-, or tri-methylated to provide additional layers of regulation. For instance, the Histone methyltransferase (HMT) Set7/9 is restricted to mono-methylation of H3-K4 (4), whereas the Drosophila trithorax (trx) and its mammalian homolog, mixed lineage leukemia (MLL) encodes a tri-methylase that is specific for $\mathrm{H} 3-\mathrm{K} 4$ (5). A recently

Abbreviations: Dnmt, DNA methyltransferase; HMT, histone methyltransferase; HDAC, histone deacetylase; HAT, histone acetyltransferase; MLL, mixed lineage leukemia; PcG, polycomb group; REST, RE1 silencing transcription factor; Trx, trithorax 


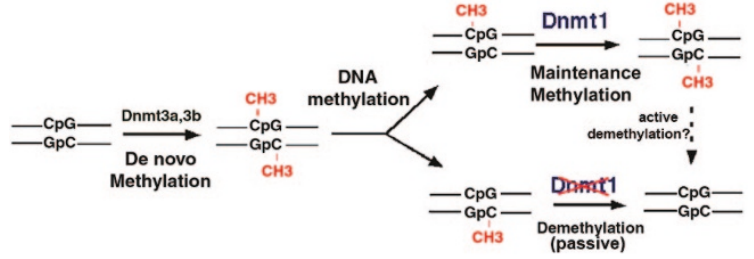

Figure 1. A schematic of the actions of the two de novo DNA methyltransferases, Dnmt3a and Dnmt3b, and the maintenance DNA methyltransferase, Dnmt1, in establishing the DNA methylation patterns and maintaining them during cell division.

discovered demethylase, LSD1 (also known as BHC110), has been shown to remove a methyl group from di- or monomethylated H3-K4 (6), which further demonstrates the dynamic nature of this epigenetic regulation. Methylation of either lysine 9 or lysine 27 on histone H3 (H3-K9 or H3-K27) is a hallmark of silent chromatin regions and is predominantly associated with heterochromatic regions and/or inactive promoters. H3-K9 HMTs, G9a and Suv39h, are localized to euchromatic and heterochromatic regions respectively (7-9). Suv39h can only methylate H3-K9 when H3 tail is unmethylated at $\mathrm{K} 4$, whereas $\mathrm{G} 9 \mathrm{a}$ can methylate $\mathrm{H} 3-\mathrm{K} 9$ regardless of the methylation status of H3-K4 (10). In addition, evolutionarily conserved multiprotein complexes, including polycomb repressive complex 2 (PRC2), are found to contain both HDAC and HMT activities, which link hypoacetylation and H3-K9/K27 methylation (11). Collectively, the existence of opposing chromatin modifying enzymes and cross-talk between different epigenetic systems demonstrate the inherent complexity in the dynamic gene regulation by epigenetic modifications (Fig. 2).

Pre- and post-transcriptional gene regulation by small non-coding RNAs. Non-coding regulatory RNA mediated regulation of gene expression is a newly emerging epigenetic mechanism. A group of these small RNAs, distinct from, but related to, siRNA (small interference RNA), are called microRNAs (miRNAs). MiRNAs are transcribed as parts of longer RNA molecules by RNA polymerase II, and then processed in the nucleus into hairpin RNAs of 70-100nts by the double stranded (ds) RNA specific ribonuclease, Drosha. The hairpin RNAs are transported to the cytoplasm via exportin-5 dependent mechanisms where they are digested by a second, dsRNA-specific ribonuclease called Dicer. The resulting 19-23 mer miRNA is bound by a complex that is similar to the RNA-Induced Silencing Complex (RISC) that participates in the siRNA pathway. In animals, the complex-bound, single-stranded miRNA binds to specific mRNAs through sequences that are significantly, although not completely, complementary to the mRNA. Via a mechanism that is still

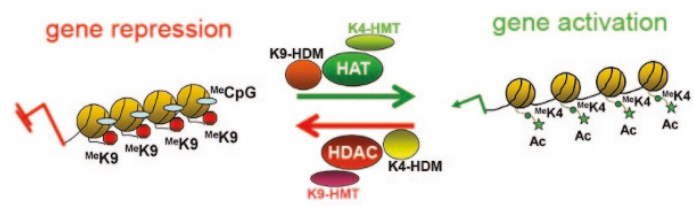

Figure 2. A schematic of histone modifications during gene activation and repression. K9-HMT: histone H3 lysine (K) 9 methyltransferase; K4-HMT: histone H3 K4 methyltransferase; K9-HDM: histone H3 K9 histone demethylase; K4-HDM: histone H3 K4 histone demethylase. not fully characterized, but which does not involve mRNA degradation, the bound mRNA does not get translated, resulting in reduced expression of the corresponding gene $(12,13)$. Recently, endogenous, small non-coding RNAs have been shown not only to regulate the stability and translation of mRNAs but also to induce the formation of inactive chromatin structures of targeted genomic sequences $(14,15)$ (Fig. 3).

\section{DNA METHYLATION AND HISTONE MODIFICATION IN STEM CELL SELF-RENEWAL AND DIFFERENTIATION}

In both the undifferentiated stem cell and their differentiated progeny, the epigenetic state is considered to be relatively stable due to its heritable nature and positive reinforcement between the multiple epigenetic mechanisms. Importantly, stable repression of genes related to terminal differentiation is absolutely required for maintenance of the stem cell pool. However, recent advances have suggested that stable and heritable epigenetic marks are reversible and dynamically regulated by opposing chromatin modifying activities, which are stimulated by extracellular differentiation-inducing cues during stem cell differentiation.

Global epigenetic reprogramming during early embryogenesis. In differentiated cells DNA methylation patterns are thought to be stable and heritable. Such a DNA methylation pattern is established gradually during several critical stages in early embryogenesis. Upon fertilization, the paternal genome is actively demethylated before the first round of cell division takes place. However, the identity of the DNA demethylase(s) underlying this active demethylation process remains elusive. After the zygote starts to divide, the passive demethylation of both paternal and maternal genomes occurs because maintenance DNA methyltransferase Dnmt1 is excluded from the nucleus (16). In the developing pre-implantation embryos de novo DNA methyltransferases begin to re-establish DNA methylation patterns during implantation and subsequent germ layer and cell type differentiation (17). This epigenetic reprogramming process ensures the erasure of acquired epigenetic information during gametogenesis. It also resets the epigenome of the pluripotent stem cells in the inner cell mass (ICM) to provide broad developmental potential (16). Indeed, many cloned embryos fail to develop due to incomplete epige-

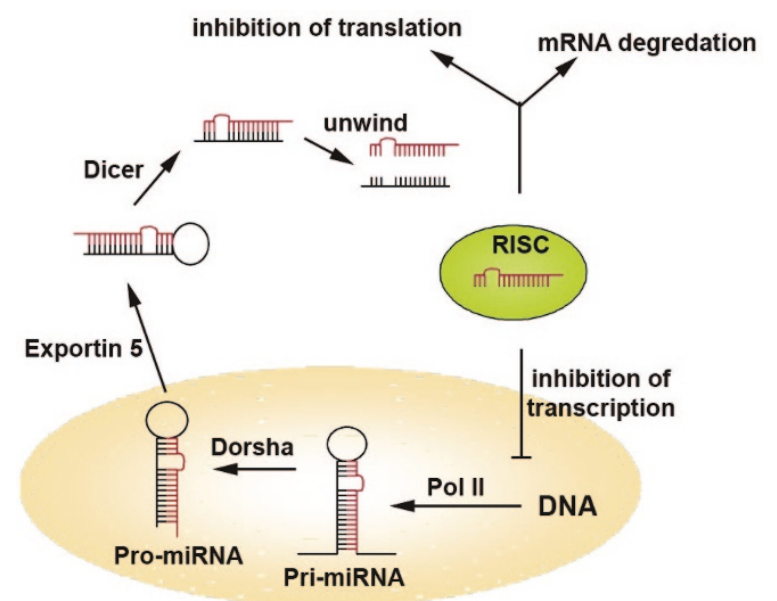

Figure 3. A schematic of the biogenesis and function of microRNAs. 
netic reprogramming (18). Furthermore, deficiency of Dnmt1 or Dnmt3 in the mouse leads to genome-wide DNA hypomethylation and early embryonic lethality (2). This suggests that DNA methyltransferase-mediated epigenetic reprogramming is required for normal development of the organism.

It has been shown that Dnmt3a is indispensable for establishing parental-specific methylation of imprinting genes during gametogenesis $(19,20)$. In addition, mutations in $D N M T 3 B$, result in human ICF (immunodeficiency, centromeric instability, facial anomalies) syndrome (17). However, little is known about how Dnmt3a and Dnmt3b are involved in establishing the epigenetic marks that account for somatic cell lineage differentiation during early embryogenesis.

DNA methylation and histone modifications in ESC self renewal. Mouse or human ESCs can be isolated from the inner cell mass (ICM) of developing blastocysts and cultured in vitro. Differentiation of stem cells is tightly regulated by interactions between extrinsic differentiation signals and intrinsic pathways. Since stem cells have extensive self-renewal capacity, the repression of differentiation genes in undifferentiated stem cells must be stable and heritable during cell division. Pluripotent ESCs contain high levels of epigenetic reprogramming activities, e.g. de novo methyltransferases and histone methyltransferases (HMTs), making ESCs excellent candidates for analysis of epigenetic reprogramming during in vitro lineage-specific differentiation. Surprisingly, both Dnmt1 ${ }^{-/-}$and Dnmt $3 a^{-/-}$; Dnmt $3 b^{-/-}$double mutant mouse ESCs are able to proliferate when maintained as undifferentiated stem cells, indicating selfrenewal capability is preserved in ESCs with hypomethylated genomes. However, these mutant ESCs fail to undergo in vitro differentiation due to either extensive apoptosis upon differentiation as a result of Dnmt1 deficiency or failure to repress pluripotent genes such as Oct4 and Nanog because of deficiency in both Dnmt3a and Dnmt3b (21). It is likely that while DNA methylation could be critically involved in silencing alternative lineage differentiation genes in tissue-specific stem cells and/or differentiated cells, histone modification-mediated gene inactivation are mainly involved in the maintenance of the self-renewal property and silencing of lineage differentiation genes in ESCs via pluripotency-related transcription factors, such as the Oct4Nanog-Sox2 complex (22).

PcG complexes are a class of histone modification enzymes, which are highly conserved throughout evolution (11). Multiprotein polycomb repressive complex 2 (PRC2), contains both HDAC and HMT activities and is involved in the initiation of gene silencing. PRC1, on the other hand, recognizes the H3-K27 methylation mark established by PRC2 through its conserved chromodomain and is implicated in stable maintenance of $\mathrm{PcG}$ complexes-mediated gene silencing effects. Bmil encodes a subunit of the PRC1 complex and Bmil-deficient mice display a defect in postnatal self renewal of both hematopoietic and neural stem cells $(23,24)$. Notably, Bmil is regulated by the Sonic hedgehog (Shh) signaling pathway in the cerebellum, providing a link between PcG and a signaling pathway that has been shown to be important for proliferation of adult neural stem cells $(25,26)$. It is likely that Bmil-associated PRC1 complexes regulate adult stem cell self-renewal through repression of the Ink4a/ Arf locus in vivo (11). Epigenetic control mediated through PcG repressor complexes is also subject to dynamic regulation from opposing chromatin modifying activities. Recently, testis specific TAF (TBP-associated factor) associated trithorax (trx) action (tri-methylation of H3-K4) was shown to counteract PcGmediated repression to allow terminal differentiation of Drosophila male germ cell precursors (27).

DNA methylation and histone modifications in stem cell differentiation along the neural lineage. Dynamic and coordinated epigenetic regulation is best illustrated by the sequential neural lineage differentiation in which neurogenesis always precedes gliogenesis during brain development. As multipotent neural stem/progenitor cells (NPCs) first undergo neuronal differentiation, early neuronal lineage genes are activated, while genes required for alternative glial fates are silenced. When NPCs enter the gliogenic phase, glial lineage genes are de-repressed in response to extrinsic cues and/or changes in intrinsic properties of stem/progenitor cells (Fig. 4). Many neuronal genes (e.g. Mashl, $B d n f$, Calbindin) contain the RE1 site within their promoters and are inhibited by REST (RE1 silencing transcription factor, or NRSF) in stem/progenitor cells and non-neuroectodermal lineages. It remains to be determined how low levels of REST proteins function to silence neuronal lineage genes in undifferentiated neurogenic NPCs. It has been suggested that the repression of neuronal genes in non-neuroectodermal lineage cells is mediated by the REST-associated $\mathrm{HDAC} / \mathrm{mSin} 3 \mathrm{~A} / \mathrm{MeCP} 2 \mathrm{com}$ plex, which leads to a hypoacetylated and subsequently H3K9 dior tri-methylated chromatin structure with high levels of DNA methylation (28). Unlike in non-neural lineage cells, where the promoters of neuronal genes are permanently silenced, the inactive neuronal promoters within stem/progenitor cells are in a

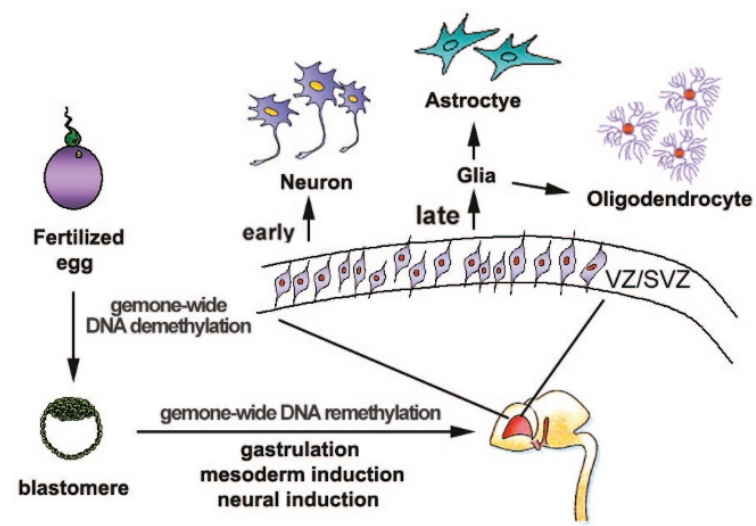

Figure 4. Genetic reprogramming during early embryogenesis and sequential neuronal versus glial lineage differentiation within the neuroepithelial progenitor zone (ventricular zone/sub-ventricular zone, VZ/SVZ) of the developing CNS (CNS).
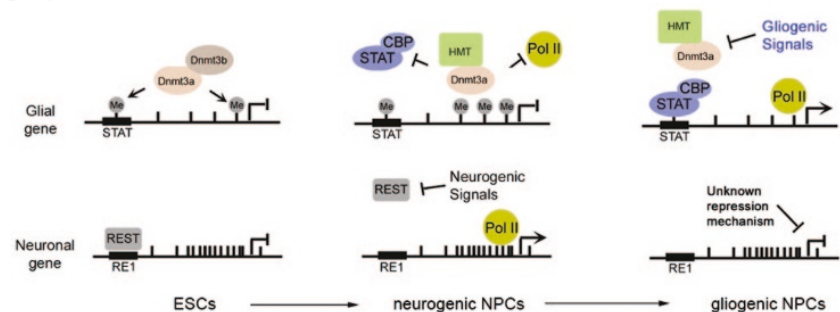

Figure 5. A model for the epigenetic control of the sequential activation of neuronal and glial genes during mouse or human ES cell differentiation along the neural lineage. 
poised state. In this state, they are not associated with DNA methylation and histone H3-K9 methylation, and once NPCs are committed to a neuronal fate and undergo terminal differentiation, the expression of REST shuts off and the REST repressor complex is dismissed from the RE1 sites within neuronal gene promoters, resulting in transcription activation. Recently, an inhibitor of BRAF35 (iBRAF) was found to activate REST controlled neuronal genes by recruiting the H3-K4 tri-methylase MLL during neuronal differentiation of P19 cells (29). Therefore, it is possible that REST may also interact with the CoREST/ LSD1 complex, which has histone $\mathrm{H} 3-\mathrm{K} 4$ demethylation activity capable of repressing neuronal gene promoters in the stem/ progenitor cells before neurogenesis.

Recent development of mouse and human ESC culture technology make it possible to derive nearly homogeneous populations of multipotent tissue-specific stem/progenitor cells from ESCs (30-32). This enables researchers to analyze the epigenetic regulations of ESC differentiation using mutant mouse ESCs deficient in various epigenetic machineries. Our recent studies analyzed the association of DNA methyltransferases and DNA methylation patterns within neuronal and glial promoters in wild-type, Dnmt $3 a^{-1-}$, and Dnmt $3 b^{-1-}$ mouse ESCs and ESC-derived NPCs. De novo methyltransferases were found to stably associate with and methylate glial, but not early neuronal lineage gene promoters in mouse ESCs as well as during the transition of ESCs to NPCs. As a result, gliogenic genes became hypermethylated and associated with an inactive chromatin structure, while early neurogenic genes remained hypomethylated and poised for transcription before and during the differentiation of ESCs along the neural lineage (Wu and Sun, unpublished observations). The different epigenetic states in early (neuronal) versus late (glial) neural lineage genes may underlie the initial changes occurring at the chromatin level that are required for pluripotent ESC differentiation toward the neural lineage and which dictate that neurogenesis precede gliogenesis (Fig. 5).

Interestingly, although both Dnmt3a and Dnmt3b are highly expressed in mouse and human ESCs, we found Dnmt3a is the predominant de novo DNA methyltransferase in NPCs derived either from ESCs or the developing mouse brain. Loss of Dnmt3a in NPCs specifically results in dysregulation of repressive epigenetic marks, including DNA methylation and histone modifications, on glial promoters, and precocious gliogenesis both in vitro and in vivo (Wu and Sun, unpublished observations). Together, these findings suggest that de novo DNA methyltransferases are indeed involved in establishing somatic, cell-type specific DNA methylation patterns during early embryogenesis. Furthermore, it is likely that preferential targeting of lineage specific genes by the de novo DNA methylation machinery is essential for proper epigenetic reprogramming, which allows for the temporally ordered activation of specific lineage differentiation programs.

Since multipotent NPCs eventually become gliogenic, the repressive epigenetic marks on glial gene promoters must not be causing permanent gene silencing. When multipotent NPCs become gliogenic, an extracellular gliogenic factor, leukemia inhibitory factor (LIF), leads to transcriptional activation and local epigenetic alterations of the proximal promoter of an astroglial gene, Gfap. These epigenetic alterations include DNA demethylation as well as concomitant chromatin remodeling consisting of a decrease in di-methylation of H3-K9 and an increase in histone acetylation. This promoter-specific epigenetic reprogramming is mediated by dissociation of the Dnmt3a with the histone modification enzyme complex and recruitment of the STAT3/ CBP transcription activator complex that contains HAT activity upon LIF stimulation (Fig. 5). It was previously reported that cytosine methylation of the $\mathrm{CpG}$ site within the STAT1/3 binding site of the Gfap promoter inhibited STAT1/3 association and therefore inhibited glial differentiation (33). However, many of the astroglial differentiation-related genes including $S 100 \beta$ and genes in the JAK-STAT pathway, do not have STAT1/3 binding elements containing embedded $\mathrm{CpG}$ sites $(34,35)$. Therefore, direct inhibition of STAT1/3 binding via methylation of the STAT binding cis-element is not a major mechanism mediating DNA methylation-related silencing of the astroglial differentiation program.

\section{REGULATION OF STEM/PROGENITOR CELL DIFFERENTIATION BY NON-CODING RNAS}

Non-coding regulatory RNA mediated epigenetic regulation of gene expression is emerging as a new focus in stem cell biology. Endogenous small regulatory RNAs can either regulate the expression of complementary mRNAs or induce formation of inactive chromatin structures of targeted genes. Expression profiling identified a subset of miRNAs that are specifically expressed in pluripotent mouse and human ESCs, suggesting a role for miRNAs to maintain undifferentiated states of stem cells $(36,37)$. Confirmation of the direct involvement of a microRNA pathway in regulation of stem cell division came from the analysis of germline stem cell (GSC) division in a Drosophila mutant for dicer-1, the RNase III essential for microRNA biogenesis (38). In mammalian cells, two c-Myc regulated microRNAs miR-17-5p and miR-20a were shown to regulate E2F1 expression, suggesting a role in regulation of cell proliferation (39).

Many microRNAs are expressed in a highly tissue-specific manner at different developmental stages (40). Recent advances suggest that tissue-specific microRNAs play a critical role in regulating cell-type specific fate choice and animal development. For example, a Notch signaling activated microRNA miR-61 regulates fate choices of vulval precursor cells in C. elegans. In mammals, overexpression of miR-181 in bone-marrow hematopoietic progenitor cells specifically increases the number of B cells (41). More recently, miR-1 was found to be specifically expressed in cardiac and skeletal muscle precursor cells. It was found that the transcription factor Hand 2 is a direct target of miR-1 mediated translational repression and that excess miR-1 leads to a decreased pool of proliferating ventricular cardiomyocytes (42). In addition to translational inhibition, developmentally regulated microRNA can also repress their targets through mRNA cleavage. The miR-196 microRNA sequences show perfect complementation to sites in the $3^{\prime}$ UTRs of the $H O X B 8$ gene (43). It was recently reported that miR-196 mediated repression of HOXB8 plays a role in limb development (44). 
The abundance of microRNAs in the postnatal brain suggests an important role for them in neuronal function. Recently, a CREB-induced microRNA miR-132 was shown to regulate neuronal morphogenesis in cultured cortical neurons (45). Surprisingly, a mutation in the $3^{\prime}$ UTR of SLITRK1, a gene involved in neurite outgrowth and implicated in Tourette's syndrome, perturbs the natural interaction between miR-189 and the 3'UTR sequences. These data suggest a role that misregulation of miR189 may be involved in this debilitating developmental neuropsychiatric disorder (46).

\section{CONCLUSION}

The epigenetic basis of stem cell differentiation arises from the need to maintain gene expression patterns in both stem/ progenitor cells and their differentiated progenies. As a stem cell differentiates, genes associated with self-renewal are down-regulated, while lineage-specific genes are activated. It then follows that the inherited epigenetic marks deposited on those genes must be reversible. Chromatin modifying enzymes with opposing activities play a key role in the dynamic regulation of epigenetic marks. Defining the signaling pathways of these enzymes induced by differentiation-inducing cues is critical to understanding the mechanisms underlying stem cell differentiation. Furthermore, non-coding RNAs, especially microRNAs, provide additional layers for regulation of gene expression during cell fate specification. These multiple layers of regulation allow for rapid transition of proliferating stem cells into their differentiated progenies.

\section{REFERENCES}

1. Jaenisch R, Bird A 2003 Epigenetic regulation of gene expression: how the genome integrates intrinsic and environmental signals. Nat Genet 33:245-254

2. Li E 2002 Chromatin modification and epigenetic reprogramming in mammalian development. Nat Rev Genet 3:662-673

3. Jenuwein T, Allis CD 2001 Translating the histone code. Science 293:1074-1080

4. Xiao B, Jing C, Wilson JR, Walker PA, Vasisht N, Kelly G, Howell S, Taylor IA, Blackburn GM, Gamblin SJ 2003 Structure and catalytic mechanism of the human histone methyltransferase SET7/9. Nature 421:652-656

5. Peterson CL, Laniel MA 2004 Histones and histone modifications. Curr Biol 14:R546-R551

6. Shi Y, Lan F, Matson C, Mulligan P, Whetstine JR, Cole PA, Casero RA, Shi Y 2004 Histone demethylation mediated by the nuclear amine oxidase homolog LSD1. Cell 119:941-953

7. Peters AH, Kubicek S, Mechtler K, O’Sullivan RJ, Derijck AA, Perez-Burgos L, Kohlmaier A, Opravil S, Tachibana M, Shinkai Y, Martens JH, Jenuwein T 2003 Partitioning and plasticity of repressive histone methylation states in mammalian chromatin. Mol Cell 12:1577-1589

8. Rice JC, Briggs SD, Ueberheide B, Barber CM, Shabanowitz J, Hunt DF, Shinkai Y, Allis CD 2003 Histone methyltransferases direct different degrees of methylation to define distinct chromatin domains. Mol Cell 12:1591-1598

9. Tachibana M, Ueda J, Fukuda M, Takeda N, Ohta T, Iwanari H, Sakihama T, Kodama T, Hamakubo T, Shinkai Y 2005 Histone methyltransferases G9a and GLP form heteromeric complexes and are both crucial for methylation of euchromatin at H3-K9. Genes Dev 19:815-826

10. Nishioka K, Chuikov S, Sarma K, Erdjument-Bromage H, Allis CD, Tempst P, Reinberg D 2002 Set9, a novel histone H3 methyltransferase that facilitates transcription by precluding histone tail modifications required for heterochromatin formation. Genes Dev 16:479-489

11. Valk-Lingbeek ME, Bruggeman SW, van Lohuizen M 2004 Stem cells and cancer; the polycomb connection. Cell 118:409-418

12. He L, Hannon GJ 2004 MicroRNAs: small RNAs with a big role in gene regulation. Nat Rev Genet 5:522-531

13. Zamore PD, Haley B 2005 Ribo-gnome: the big world of small RNAs. Science 309:1519-1524

14. Almeida R, Allshire RC 2005 RNA silencing and genome regulation. Trends Cell Biol 15:251-258

15. Bartel DP 2004 MicroRNAs: genomics, biogenesis, mechanism, and function. Cell 116:281-297

16. Reik W, Dean W, Walter J 2001 Epigenetic reprogramming in mammalian development. Science 293:1089-1093

17. Okano M, Bell DW, Haber DA, Li E 1999 DNA methyltransferases Dnmt3a and Dnmt3b are essential for de novo methylation and mammalian development. Cell 99:247-257
18. Jaenisch R, Hochedlinger K, Eggan K 2005 Nuclear cloning, epigenetic reprogramming and cellular differentiation. Novartis Found Symp. 265:107-128

19. Kaneda M, Okano M, Hata K, Sado T, Tsujimoto N, Li E, Sasaki H 2004 Essential role for de novo DNA methyltransferase Dnmt3a in paternal and maternal imprinting. Nature 429:900-903

20. Hata K, Okano M, Lei H, Li E 2002 Dnmt3L cooperates with the Dnmt3 family of de novo DNA methyltransferases to establish maternal imprints in mice. Development 129:1983-1993

21. Jackson M, Krassowska A, Gilbert N, Chevassut T, Forrester L, Ansell J, Ramsahoye B 2004 Severe global DNA hypomethylation blocks differentiation and induces histone hyperacetylation in embryonic stem cells. Mol Cell Biol 24:8862-8871

22. Boyer LA, Lee TI, Cole MF, Johnstone SE, Levine SS, Zucker JP, Guenther MG, Kumar RM, Murray HL, Jenner RG, Gifford DK, Melton DA, Jaenisch R, Young RA 2005 Core transcriptional regulatory circuitry in human embryonic stem cells. Cell 122:947-956

23. Molofsky AV, Pardal R, Iwashita T, Park IK, Clarke MF, Morrison SJ 2003 Bmi-1 dependence distinguishes neural stem cell self-renewal from progenitor proliferation. Nature 425:962-967

24. Park IK, Qian D, Kiel M, Becker MW, Pihalja M, Weissman IL, Morrison SJ, Clarke MF 2003 Bmi-1 is required for maintenance of adult self-renewing haematopoietic stem cells. Nature 423:302-305

25. Ahn S, Joyner AL 2005 In vivo analysis of quiescent adult neural stem cells responding to Sonic hedgehog. Nature 437:894-897

26. Leung C, Lingbeek M, Shakhova O, Liu J, Tanger E, Saremaslani P, Van Lohuizen M, Marino S 2004 Bmi1 is essential for cerebellar development and is overexpressed in human medulloblastomas. Nature 428:337-341

27. Chen X, Hiller M, Sancak Y, Fuller MT 2005 Tissue-specific TAFs counteract Polycomb to turn on terminal differentiation. Science 310:869-872

28. Ballas N, Grunseich C, Lu DD, Speh JC, Mandel G 2005 REST and its corepressors mediate plasticity of neuronal gene chromatin throughout neurogenesis. Cell 121:645-657

29. Wynder C, Hakimi MA, Epstein JA, Shilatifard A, Shiekhattar R 2005 Recruitment of MLL by HMG-domain protein iBRAF promotes neural differentiation. Nat Cell Biol 9:(Epub ahead of print)

30. Ying QL, Stavridis M, Griffiths D, Li M, Smith A 2003 Conversion of embryonic stem cells into neuroectodermal precursors in adherent monoculture. Nat Biotechnol 21:183-186

31. Gerrard L, Rodgers L, Cui W 2005 Differentiation of human embryonic stem cells to neural lineages in adherent culture by blocking bone morphogenetic protein signaling. Stem Cells 23:1234-1241

32. Li XJ, Du ZW, Zarnowska ED, Pankratz M, Hansen LO, Pearce RA, Zhang SC 2005 Specification of motoneurons from human embryonic stem cells. Nat Biotechnol 23:215-221

33. Takizawa T, Nakashima K, Namihira M, Ochiai W, Uemura A, Yanagisawa M, Fujita N, Nakao M, Taga T 2001 DNA methylation is a critical cell-intrinsic determinant of astrocyte differentiation in the fetal brain. Dev Cell 1:749-758

34. Fan G, Martinowich K, Chin MH, He F, Fouse SD, Hutnick L, Hattori D, Ge W, Shen Y, Wu H, ten Hoeve J, Shuai K, Sun YE 2005 DNA methylation controls the timing of astrogliogenesis through regulation of JAK-STAT signaling. Development 132:3345-3356

35. He F, Ge W, Martinowich K, Becker-Catania S, Coskun V, Zhu W, Wu H, Castro D, Guillemot F, Fan G, de Vellis J, Sun YE 2005 A positive autoregulatory loop of Jak-STAT signaling controls the onset of astrogliogenesis. Nat Neurosci 8:616-625

36. Suh MR, Lee Y, Kim JY, Kim SK, Moon SH, Lee JY, Cha KY, Chung HM, Yoon HS, Moon SY, Kim VN, Kim KS 2004 Human embryonic stem cells express a unique set of microRNAs. Dev Biol 270:488-498

37. Houbaviy HB, Murray MF, Sharp PA 2003 Embryonic stem cell-specific MicroRNAs. Dev Cell 5:351-358

38. Hatfield SD, Shcherbata HR, Fischer KA, Nakahara K, Carthew RW, Ruohola-Baker H 2005 Stem cell division is regulated by the microRNA pathway. Nature 435:974-978

39. Mertz KJ, Trees D, Levine WC, Lewis JS, Litchfield B, Pettus KS, Morse SA, St Louis ME, Weiss JB, Schwebke J, Dickes J, Kee R, Reynolds J, Hutcheson D, Green D, Dyer I, Richwald GA, Novotny J, Weisfuse I, Goldberg M, O’Donnell JA, Knaup R 1998 Etiology of genital ulcers and prevalence of human immunodeficiency virus coinfection in 10 US cities. The Genital Ulcer Disease Surveillance Group. J Infect Dis 178:1795-1798

40. Wienholds E, Kloosterman WP, Miska E, Alvarez-Saavedra E, Berezikov E, de Bruijn E, Horvitz HR, Kauppinen S, Plasterk RH 2005 MicroRNA expression in zebrafish embryonic development. Science 309:310-311

41. Chen CZ, Li L, Lodish HF, Bartel DP 2004 MicroRNAs modulate hematopoietic lineage differentiation. Science 303:83-86

42. Zhao Y, Samal E, Srivastava D 2005 Serum response factor regulates a musclespecific microRNA that targets Hand2 during cardiogenesis. Nature 436:214-220

43. Yekta S, Shih IH, Bartel DP 2004 MicroRNA-directed cleavage of HOXB8 mRNA. Science 304:594-596

44. Hornstein E, Mansfield JH, Yekta S, Hu JK, Harfe BD, McManus MT, Baskerville S, Bartel DP, Tabin CJ 2005 The microRNA miR-196 acts upstream of Hoxb8 and Shh in limb development. Nature 438:671-674

45. Vo N, Klein ME, Varlamova O, Keller DM, Yamamoto T, Goodman RH, Impey S 2005 A cAMP-response element binding protein-induced microRNA regulates neuronal morphogenesis. Proc Natl Acad Sci U S A 102:16426-16431

46. Abelson JF, Kwan KY, O'Roak BJ, Baek DY, Stillman AA, Morgan TM, Mathews CA, Pauls DL, Rasin MR, Gunel M, Davis NR, Ercan-Sencicek AG, Guez DH, Spertus JA, Leckman JF, Dure LS, Kurlan R, Singer HS, Gilbert DL, Farhi A, Louvi A, Lifton RP, Sestan N, State MW 2005 Sequence variants in SLITRK1 are associated with Tourette's syndrome. Science 310:317-320 